\title{
A PARTIAL ORDERING DEFINED BY CERTAIN MATRICES
}

\author{
JAMES R. JACKSON ${ }^{1}$
}

The purpose of this note is to characterize a simple property of matrices which the author has found to be of interest from the point of view of a mathematical description of industrial production scheduling. ${ }^{2}$

Let $\left(p_{m n}\right)$ be an $M \times N$ matrix with real entries. For integers $m, m^{\prime}$ $\in[1, M]$, we write $m \prec{ }^{*} m^{\prime}$ (read $m$ precedes $m^{\prime}$ ) if there exists an integer $n \in[1, N]$ such that $p_{m n}<0<p_{m^{\prime} n}$. We write $m<m^{\prime}$ if there exists a sequence $m_{1}, m_{2}, \cdots, m_{k}$ of integers in the interval $[1, M]$, such that

$$
m \prec^{*} m_{1}, m_{1} \prec^{*} m_{2}, \cdots, m_{k-1} \prec^{*} m_{k}, m_{k} \prec^{*} m^{\prime} .
$$

The relation " $\prec$ " is merely the extension of " $\prec *$ " to be transitive, and for $m<m^{\prime}$ we still say simply " $m$ precedes $m^{\prime}$."

In applications it is natural to assume-as the terminology suggests-that it is impossible for any $m$ to precede itself. If $\left(p_{m n}\right)$ satisfies this assumption; that is, if no $m \in[1, M]$ satisfies $m<m$, then $\left(p_{m n}\right)$ is called noncirculatory. It is obvious that $\left(p_{m n}\right)$ is noncirculatory if and only if " $\prec$ " defines a partial ordering of the integers $1,2, \cdots, M$.

Our principal business is the establishment of the following elementary theorem.

TheOREM. Matrix $\left(p_{m n}\right)$ is noncirculatory if and only if it can be replaced, by means of interchanges of rows and interchanges of columns, by a matrix $\left(q_{m n}\right)$ with the property that if $q_{\mu \nu}>0$, then whenever $m \leqq \mu$ and $n \leqq \nu$, we have $q_{m n} \geqq 0$.

Proof of sufficiency. It is obvious that interchanges of rows and columns will not destroy the noncirculatory property, so it will be enough to see that a matrix $\left(q_{m n}\right)$, as described in the theorem, is noncirculatory. This result is established by contradiction.

Presented to the Society, September 5, 1952; received by the editors July 18, 1952.

1 This paper was written under Contract Nonr 233(02), Logistics Branch, Office of Naval Research.

2 The application referred to is a specialized linear programming, which enters into the author's unpublished mathematical model of industrial production. In essence, the columns refer to industrial processes, the rows to commodities appearing in various stages of production, and the entries of the matrices are amounts of commodites produced by processes. The statement " $m$ precedes $m^{\prime \prime}$ means that, by some sequence of processing, commodity $m$ can be transformed into, or can become a part of, commodity $m^{\prime}$. 
Suppose $m<m$. Then there exist $m_{1}, m_{2}, \cdots, m_{k}$ such that $m \prec{ }^{*} m_{1}, m_{1}{ }^{*} m_{2}, \cdots, m_{k}{ }^{*} m$; there must exist $n, n_{1}, n_{2}, \cdots, n_{k}$ such that $q_{m n}<0<q_{m_{1} n}, q_{m_{1} n_{1}}<0<q_{m_{2} n_{1}}, \cdots, q_{m_{k} n_{k}}<0<q_{m n_{k}}$. But the condition on $\left(q_{m n}\right)$ then insists that $m>m_{1}>m_{2}>\cdots>m_{k}>m$, the required contradiction.

ProOF OF NECESSITY. We noted before that if $\left(p_{m n}\right)$ is noncirculatory, then " $\prec$ " defines a partial ordering of the interval of integers $[1, M]$. Hence it is easy to construct (by induction) a one-to-one transformation $\beta:[1, M] \rightarrow[1, M]$, such that $m<m^{\prime}$ implies $\beta(m)$ $>\beta\left(m^{\prime}\right)$. Define

$$
p_{\beta(m) n}^{*}=p_{m n}, \quad m \in[1, M], \quad n \in[1, N] .
$$

If $p_{m n}<0<p_{m^{\prime} n}$, then $m<m^{\prime}$, so $\beta(m)>\beta\left(m^{\prime}\right)$. Hence it is not possible that a negative entry in $\left(p_{m n}^{*}\right)$ be directly above a positive entry; that is, the entries in each column are non-negative down to some point, and are nonpositive below that point. Under these circumstances, it is obviously possible to interchange columns of $\left(p_{m n}^{*}\right)$ in such a way as to get a matrix $\left(q_{m n}\right)$ satisfying the condition of the theorem. Since $\left(p_{m n}^{*}\right)$ was obtained by interchanging the rows of $\left(p_{m n}\right)$, this completes the proof of the theorem.

The following is an obvious consequence of the symmetry of the necessary and sufficient condition of the theorem.

COROLLARY. The transpose of a noncirculatory matrix is itself noncirculatory.

The following easy corollary is of interest, because it shows that certain matrices appearing in a large class of the applications are particularly easy to invert.

Corollary. Suppose a (necessarily square) noncirculatory matrix has one positive entry in each row and one positive entry in each column, and all its other entries are nonpositive. Then it is possible by interchanges of rows and columns to bring the matrix into a form with zeros above the main diagonal, positive entries on the main diagonal, and nonpositive entries below the main diagonal.

University of California, Los Angeles 